\title{
Cultura y políticas públicas después del diluvio. Las ciencias sociales y la refundación de la política cultural
}

\author{
Arturo Rubio Arostegui \\ Universidad Antonio de Nebrija \\ jrubioa@nebrija.es \\ Joaquim RiUs-ULLDEMOLINS \\ Universitat de València / Universidad Antonio de Nebrija \\ joaquim.rius@uv.es
}

Recibido: 29-05-2014

Aceptado: 18-02-2015

\section{Resumen}

Desde su invención en los años cincuenta, la política cultural ha sido objeto de análisis y reflexión por parte de las ciencias sociales. Inicialmente, las ciencias sociales adoptaron un punto de vista crítico sobre los objetivos y los instrumentos de las actuaciones públicas en la esfera cultural. Una actitud que fueron perdiendo para acabar legitimando de forma acrítica los proyectos de desarrollo económico y urbano basados en la cultura. Este paradigma se revela actualmente agotado a causa de una excesiva instrumentalización de la cultura y por el colapso general del sistema cultural. Un colapso generado por la crisis económica, la falta de planificación y evaluación y, la transición al paradigma digital. En este contexto, los autores plantean la necesidad de desarrollar una nueva visión multidimensional, crítica y sistemática de la política cultural. Esta debe abordar sus retos principales como la refundación de sus objetivos, sus formas de gobernanza y gestión. Asimismo, la política cultural debe replantearse su relación con las formas emergentes de creación y participación social descentralizada en la sociedad digital.

Palabras clave: política cultural, ciencias sociales, gestión pública, valor público, participación ciudadana, crisis. 


\title{
Culture and Public Policies after the Deluge. Social Sciences and the Re-Foundation of the Cultural Policies
}

\begin{abstract}
Since its invention in the fifties, cultural policy has been the subject of analysis and reflection by the social sciences. Initially, social sciences adopted a critical point of view on the objectives and instruments of public action in the cultural sphere. An attitude that Social Science were losing and finally ended uncritically legitimizing the projects of economic and urban development based on culture. This paradigm is currently exhausted, first, by the excesses one vision instrumentalization of culture and, moreover, by a general collapse of the cultural system due to the economic crisis, lack of planning and evaluation and, because of the transition to digital paradigm. In this context, the authors suggest the need to develop a multidimensional, systematic and critic review of the cultural policy, addressing its major challenges such as the rebuilding of its objectives, new forms of governance and management and its relation to emerging forms of creation and decentralized social participation in the digital society.
\end{abstract}

Keywords: Cultural Policy; Social Sciences; Public Management; Public Value; Citizen Participation; Crisis.

\section{Referencia normalizada}

Rubio Arostegui, Arturo y Joaquím Rius-Ulldemolins (2015): “Cultura y políticas públicas después del diluvio. Las ciencias sociales y la refundación de la política cultural”, Política y Sociedad, 52 (1), pp. 27-52.

Sumario: 1. Introducción. la relación entre ciencias sociales y política cultural. 2. La esfera cultural: del "diluvio" a la refundación. 3. Las políticas culturales y las ciencias sociales: entre la instrumentalización al servicio del poder y la crítica a sus efectos sociales y políticos. 4 . La esfera cultural y las ciencias sociales: investigación, crítica y evaluación. 5. Conclusiones. 6. Bibliografía. 


\section{Introducción: la relación entre ciencias sociales y política cultural}

Progresivamente en toda Europa ha ganado progresivamente fuerza la idea de la necesidad de repensar y refundar la política cultural (McGuigan, 2004; Dubois, 2010). Sin duda, en ello se refleja la crisis del rol tradicional del Estado-nación de regular, redistribuir y convertir la cultura en un factor de cohesión social (Rius, 2005; Menger, 2010), así como el creciente rol que ocupa la cultura como factor de desarrollo social y económico regional y local (García, 2004; Degen y García, 2012; Sánchez et al., 2013). Frente a esta complejidad, las políticas culturales se han apoyado en las ciencias sociales para desarrollar un paradigma más coherente. Sin embargo, la relación entre política cultural y ciencias sociales, si bien ha existido desde el nacimiento de las políticas públicas en el ámbito cultural en los años cincuenta, especialmente en países como Francia e Inglaterra (Rubio Arostegui, 2008). Es conocido que André Malraux, el intelectual y luego Ministro de Cultura, concebía la política cultural como un medio de liberar a las masas de los efectos narcotizantes de la industria cultural denunciados por Adorno y Horkheimer (Urfalino, 1996). Posteriormente, el análisis sociológico ha ejercido una notable influencia en cambiar y en mostrar los límites sociales de las políticas democratizadoras de la cultura (Bourdieu et al., 2003), en orientar la política cultural hacia una concepción más relativista de la cultura (V. Dubois, 2010) o bien en orientarla a buscar unos efectos sociales positivos e integradores (Bianchini, 1991; Bloomfield y Bianchini, 2004; Subirats et al., 2010). Sin embargo, actualmente las ciencias sociales tienen dificultades en analizar y responder a los retos que ha planteado la confluencia de diversas crisis del sector cultural (económica, sistémica y de legitimación). Una crisis que podemos calificar de un "diluvio" que amenaza la supervivencia de la esfera cultural, al menos tal y como la conocemos ahora.

Ciertamente, esta debilidad no es nueva y ya en 2009, Rubio Arostegui (2008) plantea por primera vez en España los posibles efectos del discurso de las ciencias sociales en la política cultural y en las lógicas de los campos artísticos. A partir de su análisis diacrónico y descriptivo la producción científica en el campo de las políticas culturales en España, Rubio Arostegui destaca una debilidad tanto cuantitativa como cualitativa comparada con otros países europeos (ibídem). Así, el conocimiento que se ha ido produciendo desde los másteres en gestión cultural nacidos en España a principios de la década de los noventa del pasado siglo, han sido valiosos y fundamentalmente descriptivos. Y por otra parte, los valiosos pero puntuales aportes de la sociología de las artes

${ }^{1}$ La expresión "diluvio", si bien no es un concepto científico, es utilizado comúnmente para referirse a una situación de colapso y caos, en el que las normas anteriores no rigen más. La expresión "diluvio" para referirse a una situación social suele remontarse popularmente a la frase de Madame Pompadour dirigida al Rey de Francia Luis XV “après moi, le déluge", refiriéndose a una futura situación de caos producto de una derrota militar (Cf. déluge, Dictionnaire de l'Académie française). 
a través de los primeros trabajos de Rodriguez Morató, Antonio Ariño o Xan Bouzada (2005) no han permitido crear un estudio longitudinal y sistemático de la evolución del conjunto de la política cultural en el Estado español.

Asimismo, la aportación de la consultoría cultural que en un primer momento fue generada por las administraciones públicas, posteriormente también ha sido demandada en mayor o menor medida por los stakeholders de los campos artísticos creando así un mercado del conocimiento acerca de las industrias culturales y creativas (Rubio Arostegui, op. cit.). Sin embargo, estos estudios han tenido muchas veces una función de legitimación de las reivindicaciones sectoriales que de verdadera acumulación de conocimiento sobre los sectores culturales. Al mismo tiempo, cabe destacar que a diferencia de otros campos de la política pública en los que hubo una crítica sistemática a los objetivos, los instrumentos y los efectos de las políticas públicas, el análisis de las políticas culturales en el Estado español se ha caracterizado por una debilidad en sus bases teóricas y metodológicas (Rodriguez Morató, 2012), y por un carácter muchas veces descriptivo de experiencias siempre en clave positiva o de recetario de modelos de éxito (cf. Roselló y Colombo, 2008; Manito Lorite, 2008). ${ }^{2}$

En este artículo nos planteamos ir más allá y tratar de proponer la cuestión de si las ciencias sociales, en relación con la política cultural, deben tener este nivel descriptivo y valorizador de lo existente o exitoso. O por el contrario las ciencias sociales deben ser más ambiciosas, ir más allá y adoptar un punto de vista distanciado y crítico. Nuestro punto de vista es el segundo y creemos, tal como plantea Ema López (2013), que las ciencias sociales deben apostar por esta opción, a causa de "el papel cada vez más relevante de las ciencias, y en concreto de las ciencias sociales, como dispositivo que contribuye a naturalizar el orden establecido como el único horizonte de lo posible" (op. cit.: 16). Por lo tanto, debe existir cierta producción científica que plantee en sus trabajos romper esa naturalización de la realidad artística y cultural, tanteando escenarios alternativos que replanteen o hagan reflexionar sobre lo establecido.

El diagnóstico del artículo se basa en la investigación realizada por los autores en 2014 para la Fundación Alternativas (Rubio Arostegui et al., 2014) ${ }^{3}$ a través de la me-

${ }^{2}$ En los tiempos de expansión económica y de crecimiento de la política cultural, los análisis de la política y la gestión cultural ha sido -con pocas excepciones- realizados desde una perspectiva excesivamente positiva, destacando solamente aquellos casos que desde algún punto de vista constituían un éxito. Las dos obras citadas casos citados son ejemplares de la poca distancia crítica de los análisis, al no destacar ningún caso de "fracaso", centrarse en casos de éxito desde un punto de vista artístico y/o empresarial sin analizar su relación con objetivos de valor público, la relación de coste-beneficio o sus efectos sociales a largo plazo.

${ }^{3}$ Este artículo recoge una parte del resultado del Proyecto de Investigación "El modelo de financiación de las artes y la cultura en el contexto europeo. Crisis económica, cambio institucional, gobernanza y Valor 
todología Delphi de construcción de un consenso a partir de expertos. ${ }^{4}$ A partir de este diagnóstico, el artículo trata de plantear y resituar la política cultural en un contexto social de cambio acelerado, de crisis del Estado del Bienestar y en un proceso de transición del mundo cultural hacia el paradigma digital. ${ }^{5} \mathrm{El}$ propósito de esta investigación

público de la cultura y la política cultural'. Este estudio, coordinado por Arturo Rubio (Universidad de nebrija) y por Joaquim Rius (Universitat de València), fue financiado por la Fundación Alternativas, con la colaboración de la Fundación SGAE y tuvo una duración de 8 meses, dando comienzo al 1/06/2013 hasta el 31/1/2014. El proyecto contó con un equipo de trabajo compuesto por 4 investigadores, Victoria Sánchez Belando (Universidad de Barcelona) y Santi Martinez Illa (Consultor independiente) y contó con la participación del Grupo de Investigación Nebrija de Arte (Universidad Antonio de Nebrija). La investigación fue presentada en la sede de la Fundación Alternativas en 2014 con una gran difusión mediática (Cf. El País, España es el país europeo que más ha recortado en cultura desde 2011. 26/03/2014. Disponible en: $<$ http://cultura.elpais.com/cultura/2014/03/26/actualidad/1395840976 825091.html>).

${ }^{4}$ El artículo se basa en los resultados de la metodología Delphi de la citada investigación (Rubio Arostegui et al., op. cit.) que ha consistido en dos rondas de encuesta a veinte expertos del sector cultural seleccionados. Asimismo, se convocaron dos grupos de discusión que reunieron a quince responsables del sector cultural y académicos para debatir y validar los análisis y propuestas de los expertos, uno realizado en Madrid y otro en Barcelona. Todo el proceso se ha desarrollado bajo la dirección de Arturo Rubio Arostegui y Joaquim Rius-Ulldemolins, y con la colaboración de Santi Martinez Illa y Victoria Sánchez Belando. Queremos agradecer asimismo la participación de todas las personas que han colaborado en el Delphi y en los grupos de discusión: Fátima Anllo (Universidad Complutense de Madrid), Antonio Ariño (Universitat de València), Antonio Ávila Álvarez (Federación de Gremios de Editores de España), Jordi Baltà (Interarts), Lluis Bonet (Universitat de Barcelona), Javier Brun Gonzalez (Ayuntamiento de Huesca), Ruben Caravaca (Asociación de Gestores Culturales de la Comunidad de Madrid), Jorge Fernández León (Ayuntamiento de Gijón), Roberto Gómez de la Iglesia (c2+i / Conexiones improbables), Juan Antonio Hormigón Blánquez (Asociación de Directores de Escena de España), Jorge Luis Marzo (Consultor Freelance), Angel Mestres (Trànsit Projectes), Jordi Pascual (UGLC-Agenda 21 de la cultura), Hector M. Pose Porto (Universidade da Coruña), Cristina Ramírez (Ayuntamiento de San Fernando de Henares), César Rendueles (Universidad Compluense de Madrid), Francisco Rodríguez Peinado (Asociación de Autores de Cómic de España), Yolanda Romero Gomez (Asociación de Directores de Arte Contemporáneo en España), JaronRowan (Goldsmiths University), Joan Subirats (Universitat Autònoma de Barcelona).

${ }^{5}$ Así pues, la utilización de la técnica de Delphi se justifica por la complejidad de la situación tratada y su carácter dinámico. El principal criterio que guió la selección de las personas consultadas, fue que se tratasen de referentes en el campo de la política cultural por su conocimiento experto, pero también por haber tenido una trayectoria variada como profesionales o académicos en relación al sector público, privado y asociativo. Consideramos que el aporte de expertos con este perfil en la definición de líneas de actuación y recomendaciones sería enriquecedor en relación a uno de los objetivos de este estudio: el de captar la evolución y perspectivas de la política cultural en un escenario de cambio en el que las fronteras entre las lógicas públicas de actuación y las privadas se hacen cada vez más difusas en el marco de una inestabilidad sistémica, dando lugar a nuevas formas de entender y practicar la producción, circulación y consumos culturales. Al finalizar esta etapa, los datos recogidos fueron utilizados para elaborar un segundo cuestionario. Asimismo se celebraron dos grupos de discusión, uno en Madrid (28/10/2013) y otro en Barcelona (18/11/2013). Los resultados del Delphi como de los grupos de discusión han sido transcritos y analizados su contenido de forma temática. Parte de los resultados han sido incorporados en el presente artículo, aunque sin recurrir a la reproducción literal para no sobrecargar la lectura del diagnóstico. 
era el de ampliar los horizontes de reflexión y acción. Y servir de instrumento para formular políticas culturales multi-nivel y aportar herramientas que velen por la modernización de las instituciones culturales. El concepto de transferencia del conocimiento en el marco del capitalismo cognitivo puede estar al servicio de las lógicas e intereses de los campos artísticos o de las administraciones públicas pero también puede tener fines propositivos que puedan aportar nuevas direcciones al discurso de la política cultural. Este discurso académico o científico que a trata de aportar valor a los sujetos investigados tiene precedentes históricos en la producción de Pierre Bourdieu $(1991,2002)$ y en otros enfoques metodológicos de las ciencias sociales aplicados a otros ámbitos sociales y culturales como la Investigación-acción, el construccionismo o las epistemologías feministas (Rubio Aróstegui, 2008).

En este sentido, la democratización cultural como eje básico en la génesis de las políticas culturales de los Estados en el siglo XX es en alguna medida una formulación de una política emancipadora como también lo fueron en su momento las políticas educativas de los Estados que paliaron el analfabetismo en Europa (Zimmer y Toepler, 1996; V. Dubois, 1999). Pero estos valores de las políticas que tienen su germen común en el Estado del Bienestar europeo hoy están en franca recesión. Además, el desmantelamiento acelerado del Estado del Bienestar a raíz de la crisis económico-financiera en el siglo XXI - sobre todo el sur de Europa- se puede constatar a partir de los datos estadísticos actualizados de gastocultural (Rubio Arostegui et al., 2014). Asimismo, nos encontramos ante una crisis de legitimidad de la política cultural: no es fácil hallar un acuerdo absoluto entre los académicos europeos acerca del valor de la democratización cultural, porque el estudio de sus impactos y logros muestra como estos consisten más en la intensificación de los consumos culturales en las capas medias de la sociedad que el de mejorar las tasas de participación del conjunto de la población. Así pues, no faltan los investigadores que muestran cómo los sectores con niveles sociales y educativos bajos quedan excluidos en buena medida de la esfera cultural, tal como demuestran los estudios sobre prácticas y hábitos culturales longitudinales (Poirrier, 2013).

Por lo tanto, creemos que el papel de la producción científica en torno a la política cultural debe tener un doble valor descriptivo y prescriptivo sin ambages con respecto a los valores en las que se basa la subjetividad del investigador social. Por lo tanto, cuando apostamos por el valor de la cultura y sus profesiones defendemos conceptual y empíricamente el valor público que entendemos que éstas aportan al subsistema social y cultural del país. A la vez que también consideramos la importancia del reconocimiento de la propiedad intelectual de autores y artistas de acuerdo a los nuevos tiempos del paradigma digital. Para finalizar, entendemos que el compromiso de las ciencias sociales en la defensa del derecho a la cultura, comporta también el ser extremadamente exigentes al evaluar la gestión y el impacto de las instituciones artísticas y culturales y criticar su ausencia de transparencia y cultura de rendición de cuentas. 
Así pues, el artículo se plantea las siguientes preguntas: ¿Qué pueden aportar las ciencias sociales al campo cultural? ¿Qué papel pueden jugar las ciencias sociales en el campo de la cultura en general y en particular en las reglas de los campos artísticos, la recepción cultural o la modernización y el valor público de la gestión de las instituciones artísticas? Para ello, abordaremos en primer lugar el análisis de lo que podemos describir metafóricamente como un "diluvio" sobre el sistema cultural y la necesidad de refundación que se imponga después de este "diluvio". En segundo lugar, abordaremos críticamente qué rol han desarrollado las ciencias sociales y sus aplicaciones en forma de consultoría en el devenir de la política y la gestión cultural en España. Finalmente, propondremos lo que según nuestro punto de vista y a la luz de la diagnosis de los expertos consultados en el Delphi (ver nota al pie 4 y 5 ) constituyen las futuras tareas de las ciencias sociales en relación a la política y la gestión cultural para buscar una salida alternativa al actual estado de crisis y disolución de la esfera cultural: a) La búsqueda de nuevos objetivos y legitimaciones de la política cultural, b) La crítica en la actual gobernanza de la cultura y la transformación en la forma de gestionarla y, c) La necesidad de buscar nuevas regulaciones y de replantear la participación cultural inscribiéndola en los procesos de innovación social en curso en el entorno digital y de los movimientos sociales.

\section{La esfera cultural: del "diluvio" a la refundación}

Desde los años 80 , podemos interpretar las dinámicas posmodernas como un proceso en el que la esfera cultural gana una mayor centralidad social de la cultura y a la vez ésta padece una mayor mercantilización e instrumentalización para fines económicos y sociales (Gray, 2007). En este sentido, podemos hablar de una situación paradójica: por un lado, existe un discurso sobre las industrias culturales y creativas que les asigna un rol fundamental en el desarrollo económico, social y personal (Gray, 2007) y unas prácticas que asignan a la cultura un potencial transformador de la sociedad (Belfiore y Bennett, 2007). Pero por otro lado, todos los sectores culturales, y parece que ninguno queda a salvo, se enfrentan al diluvio de la gran recesión económica, la caída de la financiación pública y una crisis de los paradigmas institucionales basados en el mercado y la intervención del Estado (V. Dubois, 2010; Levine et al., 2013). Después de tres décadas de crecimiento casi sostenido, las gráficas y estadísticas culturales se expresan en números rojos en casi todos sus indicadores: financiación pública, público, ventas, facturación oempleo (Bustamante, 2011; Rubio Arostegui et al., 2014). ${ }^{6}$ Asimismo, desde hace veinte años no han

${ }^{6}$ Según la citada investigación (Rubio Aróstegui et al., 2008) actualmente, todos los datos disponibles muestran grandes reducciones: en los presupuestos públicos dedicados a la cultura la reducción entre 2009 y 2013 ronda el 50\% (48\% en el caso del Ministerio de Educación y Cultura y alrededor del 40\% en las Comunidades Autónomas). En relación a la actividad y consumo cultural, todos los datos disponibles 
faltado las diagnosis sobre el efecto perverso de las políticas culturales al crear un sector cultural muy dependiente de las ayudas públicas y distanciado de la ciudadanía (Poirrier, 2013) y las llamadas a su reinvención desde nuevas bases (McGuigan, 2004).

Ante esta realidad, hay una aparente desconexión de lo cultural y lo político y un cuestionamiento de las instituciones, cadenas cooperativas y mecanismos de asignación de recursos, que constituyen su base organizativa e institucional y que, asimismo, apuntan a una reconfiguración de los paradigmas clásicos de producción, distribución y consumo cultural, así como de la articulación y papel entre actores estatales, del mercado y sociales en que estos se sustentan (Rubio Arostegui et al., 2014).

En España, esta crisis se refleja en la esfera cultural en: a) La disminución o desaparición de los apoyos públicos a la cultura, al ser considerada por gran parte de las élites sociales y políticas como un servicio no esencial, b) La creciente interpretación de la esfera cultural por parte de las administraciones públicas como un recurso para competir en el mercado global, ya sea para las ciudades grandes o medianas (Evans, 2003) o bien para los Estados-nación como una herramienta para configurar y promover la marca-país (Dinnie, 2008; Rius-Ulldemolins y Zamorano, 2014). Y c) La crisis de valor de la esfera cultural se caracteriza por una paupérrima valoración de la ciudadanía y de la política acerca de las profesiones culturales, su función y la protección al derecho de obtener una retribución por el trabajo creativo. ${ }^{7}$

Finalmente, continuando con la analogía de la lluvia, podemos decir que en el Estado español "llueve sobre mojado". No se trata solamente, como se afirma habitualmente, de una situación de crisis creada por la falta crónica de recursos. Ello sólo explica en parte los problemas de la política cultural. Sino que en gran parte las dificultades de la política cultural en España radican en una falta de planificación sistemática, una gobernanza multinivel y una orientación a medio y largo plazo sostenible. Unas deficiencias que a pesar de haber sido diagnosticadas no han sido corregidas en la política cultural desarrollada los treinta últimos años (Bouzada, 2008; Rius-Uldemollins y Zamorano, 2014). En efecto, podemos observar que en la última década España ha sido de los países que más ha crecido en gasto cultural aproximándose, a final de ésta, a los niveles europeos, hecho que sin embargo no ha conllevado unos niveles de consumo cultural

en Eurostat o el Compendium de políticas culturales tenderían a corroborar la hipótesis de impacto de la crisis con efectos retardados y más agudos en los países de la periferia del euro. En el caso español, las estadísticas de algunos sectores como el escénico o la música en directo presentaron balances relativamente optimistas hasta 2012 cuando a la fuerte recaída en la recesión económica y los recortes del sector público se sumó la subida del IVA cultural en una suerte de "tormenta perfecta". Una tormenta que arreciaría sobre sectores de las industrias culturales como el audiovisual o el editorial más expuestos a las inclemencias de la propia crisis y los efectos de la digitalización.

${ }^{7}$ Esta baja valoración de la cultura se refleja en los índices de piratería. España, según los informes internacionales, es uno de los países europeos donde hay un porcentaje más alto de la población que consume productos artísticos sin pagar derechos de autor (Jiménez Zaera, 2013). 
o de institucionalización de la cultura comparables a estos países de referencia (Rubio Arostegui et al., 2014). ¿ ¿Si no son sólo los medios, entonces qué es lo que ha fallado? Como veremos a continuación, las inversiones en cultura han sido instrumentalizadas por un modelo de desarrollo económico, de orden social y político al que las ciencias sociales no han sabido o no han querido reformular o combatir y que desde nuestro punto de vista ha conllevado, como veremos, su deslegitimación y posterior colapso.

\section{Las políticas culturales y las ciencias sociales: entre la instrumentalización al servicio del poder y la crítica a sus efectos sociales y políticos}

Las relaciones entre cultura y política, pasaron del enfrentamiento en la etapa de la autonomización de la esfera artística - desde el siglo XIX y parte del siglo XX-, a una intensa interrelación y dependencia en la segunda mitad del siglo XX (Bourdieu, 1977; V. Dubois, 1999). En un primer momento, la esfera cultural marcó el rumbo de la política cultural imponiendo legitimidad y valores (Urfalino, 1989). Sin embargo, a finales del siglo XX y principios del siglo XXI, se produce una transformación en la política cultural en la que los responsables políticos adquieren un mayor protagonismo en la definición de acción política pública en el ámbito cultural y del rol de las instituciones culturales (V. B. Dubois Clément et al., 2012, Rius, 2014). Esta tendencia tiene efectos positivos al corregir la tendencia a la auto-referencialidad de los sectores culturales y aportar visiones más enfocadas a satisfacer las aspiraciones de la ciudadanía, es decir, a aportar valor público (Holden, 2008). Pero llevada a un extremo puede acabar desnaturalizando la política cultural al someter las decisiones sobre programación artística a una lógica clientelar o económica, sin tener en cuenta la calidad o la innovación propiamente cultural (Gray, 2007; Gray, 2008).

Sin embargo, la creciente instrumentalización de la cultura para fines económicos, urbanos y sociales, se basa en el asumir a priori que la cultura genera impactos y efectos positivos; un discurso que desde los años ochenta ha ido sustituyendo las legitimaciones tradicionales basadas en el valor cultural (Belfiore y Bennett, 2007; Belfiore y Bennett, 2008). Un axioma que sin embargo raramente se ha basado en evidencias empíricas constatables y sí en el desarrollo de un discurso por parte de los dirigentes políticos en

${ }^{8}$ España ha sido el tercer país de la Unión Europea en el que más han crecido los presupuestos culturales: desde el año 2000 al año 2010 el presupuesto cultural ha aumentado un 53\% en euros constantes, situándose en un gasto per cápita el 2010 en 149 euros, muy por encima de la media de la UE-15 situada en 101 euros (Rubio y Rius, 2014). Sin duda podemos escudarnos en los factores macroestructurales heredados del franquismo y la Transición para explicar las deficiencias de la política cultural (Fishman y Lizardo, 2013). Sin embargo para los autores, estos factores aún teniendo su peso explicativo, no justifican porqué en este momento la política cultural se halla en semejante situación al borde del colapso. 
alianza con consultorías privadas (Belfiore, 2002; Belfiore, 2004). Este cambio programático y discursivo en la política cultural no se ha desarrollado en el seno de los ámbitos deliberativos de la cultura como los consejos de las artes o los Ministerios de Cultura, sino que principalmente lo han gestado y difundido las élites políticas y económicas a partir de los medios de comunicación, con el soporte técnico en marketing y comunicación de las consultorías privadas. En efecto, desde su inicio a mediados de los años ochenta, los think tank y las consultoras culturales se han constituido como elaboradoras y legitimadoras de las nuevas políticas culturales; que partiendo del neoliberalismo y su giro emprendedor, fomentan que los gobiernos locales presenten a la cultura como una forma "innovadora" de resolver los problemas sociales sin aumentar los gastos sociales o sin replantear las políticas económicas (Mooney, 2004; Miles, 2005).

Estas consultorías, surgidas al calor del Nuevo laborismo británico, acompañan el giro de las políticas culturales hacia una política de apoyo de las llamadas industrias creativas y al fomento de las empresas creativas y el management cultural (Bilton, 2007; Schlesinger, 2013). Y actualmente estos discursos se han difundido en la mayoría de países a través de los medios académicos y de las consultorías privadas internacionales asociando la idea de la cultura como instrumento de desarrollo económico y fomento de la emprendiduría, que según esta visión aporta la creación cultural y artística (Cunningham, 2009). ${ }^{9}$ En relación con la emprendiduría esta se ha convertido en una ideología omnicomprensiva del sector cultural (Rowan, 2010), que atribuye al sector cultural un rol instrumental a todo un sector económico o vector económico de desarrollo (las llamadas industrias creativas). Esta tendencia desarticula un poco más la autonomía de la esfera artística al convertirla en una actitud psicológica (la creatividad como competencia y habilidad). Una redefinición que la subsume a una gran diversidad y heterogeneidad de tareas humanas y que la sitúa al margen de su tradición humanística y disciplinar. Esta concepción sitúa la creación cultural en un registro, el de la gestión de la creatividad, dominado por los consultores y los think tanks (Fullerton y Ettema, 2014). Producto de ello es la confusión entre las políticas culturales y las de promoción económica o de las actividades culturales con las de capacitación de competencias y habilidades (Jones, 2010).

Otro de los discursos dominantes a nivel internacional ha sido el de la ciudad creativa, iniciado por Landry y Bianchini (1995) y desarrollado por Richard Florida con su defensa -más normativa que analítica- de la capacidad de las clases creativas para transformar la economía urbana (Florida, 2005). Una idea criticada por su inconsistencia teórica en el uso del concepto de clase social y por sus efectos gentrificadores de las ciudades en las que se ha aplicado (Peck, 2005; Pratt, 2008). A pesar de estas críticas, este discurso ha

${ }^{9}$ España, y especialmente Barcelona, ha sido uno de los lugares europeos donde este discurso y su forma de operar ha gozado de mayor éxito, convirtiéndose en uno de los elementos del llamado "Modelo Barcelona" de política cultural (Degen y García, 2012; Sánchez et al., 2013). 
contado con un notable prestigio y difusión en el Estado español, en el que se han desarrollado sucesivos encuentros de gobernantes, consultoras y agentes culturales para intercambiar experiencias y en general promover este paradigma (cf. Manito Lorite, 2010).

El modelo de ciudades creativas ha encajado además de forma notable en España por el protagonismo cultural de las ciudades, la importancia del turismo y la tendencia a la construcción de infraestructuras culturales sobredimensionadas, legitimadoras del poder político, sea este estatal, autonómico o local (Rubio y Rius, 2012; Rius, 2014). En algunas ciudades, este paradigma de la ciudad creativa ha conllevado la celebración de costosos eventos, como el Fórum de las Culturas de Barcelona 2004 sin un retorno social y económico claro, excepto para las empresas constructoras y los especuladores inmobiliarios (Majoor, 2011). Asimismo, esta ideología promovida y legitimada por las consultorías ha conllevado también volcarse en la construcción de grandes infraestructuras culturales que a menudo han sido mal diseñadas respecto a sus usos futuros y las necesidades culturales locales, como es el caso de la Ciudad de las Artes en València, la Ciudad de la Cultura o las costosas ampliaciones de las infraestructuras de la capital del Estado, como el Museo Reina Sofía (Hernàndez i Martí y Albert Rodrigo, 2012; Lage et al., 2012, Rius-Ulldemolins et al., 2012). Estas infraestructuras, además de representar un enorme gasto de inversión y una hipoteca en relación a los futuros presupuestos por su elevado coste de mantenimiento, han supuesto un enorme coste de oportunidad al dirigir todas las energías del sector cultural en promoverlas (o criticarlas), dejando así de lado los esfuerzos por tejer verdaderos sistemas para la política cultural estructurados y espacios de participación cultural verdaderamente sostenibles (Sánchez et al., 2013). ${ }^{10}$

Asimismo, todos estos efectos perversos eran previsibles a causa de la falta de planificación en función de necesidades culturales objetivas y su poca orientación a la sostenibilidad social y cultural (Martinez y Rius, 2010, Kagan y Hahn, 2011). Sin embargo, pocas fueron las voces desde las ciencias sociales que se alzaron contra estos procesos, que han convertido a las políticas culturales, en subsidiarias de la construcción de marcas urbanas internacionales (Pike, 2011; Rius-Ulldemolins y Zamorano, 2014).

Precisamente, en el área de los estudios urbanos es donde han surgido las críticas más radicales a este modelo, señalando sus efectos mercantilizadores sobre los espacios urbanos y la desarticulación de las relaciones comunitarias (Balibrea, 2004; Delgado, 2008). No obstante, esta crítica no ha conseguido influir en la planificación y toma de decisiones de la gestión pública de la cultura. Muestra de ello es que hasta 2010, momento en el que se caminaba al borde del abismo ciudadano, político y social,

${ }^{10}$ Uno de los casos más llamativos de la distancia entre la inversión realizada en cultura y el retorno social y cultural de la inversión es el caso del País Valenciano: 1.200 millones enterrados en esa Ciudad de las Artes y las Ciencias unas instalaciones tan caras como infrautilizadas, generaron un déficit en 2013 de 52 millones. Por otra parte, más de 300 millones costó la Ciudad de la Luz, un megalómano estudio de cine que desde 2012 ha cerrado sus puertas por falta de viabilidad económica (Hernàndez i Martí y Albert Rodrigo, 2012). 
muchos consultores y académicos continuaban promoviendo proyectos de clústeres de industrias culturales de cuantiosas inversiones e hipotéticos rendimientos futuros, como por ejemplo el proyecto del clúster audiovisual en la Zona Franca de Barcelona. ${ }^{11}$

\section{La esfera cultural y las ciencias sociales: investigación, crítica y evaluación}

Las ciencias sociales deben por lo tanto dejar de ser legitimadoras del giro neoliberal de las políticas culturales o utilizar los argumentos de la economía y la sociología para justificar los proyectos del poder. Al contrario, deben re-focalizarse en estudiar la eficacia, la eficiencia y el impacto de los mecanismos propios de la política cultural. Tres son a nuestro entender los grandes retos de la política cultural: 1) Reformular el valor público y la financiación de las artes en el marco internacional, 2) Acompañar la modernización de la gestión y la gobernanza de la cultura y, 3) Actualizar y buscar consensos en relación entre la industria cultural y los movimientos sociales en torno a los derechos de autor y la creación digital.

\subsection{Reformular el valor público y la financiación de las artes en el marco internacional}

La política cultural como proyecto de democratización cultural ha ido perdiendo sentido en el marco nacional por la llegada de nuevos paradigmas de consumo cultural, especialmente en el ámbito digital (Ariño Villarroya, 2010). Asimismo, la política cultural ya no puede seguirse comprendiendo sólo desde una perspectiva nacional ya que desde los años setenta van ganando cada vez más importancia otros agentes cada vez más relevantes (Menger, 2010). Unos agentes actúan desde el interior del Estado-nación, los gobiernos regionales y los gobiernos locales convirtiéndose en los agentes estructuradores

${ }^{11}$ Algunos podrían pensar que el sonoro fracaso de muchas iniciativas que prometían convertir la cultura en el nuevo motor de la economía y en atracción de los turistas internacionales, como es el caso del Fòrum de les Cultures 2004 de Barcelona o la Ciutat de les Arts i les Ciències de Valencia por poner dos casos, debería hacer reflexionar a los responsables de la política cultural en los límites y los efectos no deseados de tal orientación. Pero parece que de momento no es así: hasta 2012 se han seguido elaborando proyectos bajo el paradigma de la ciudad creativa como el de la BZ Barcelona Zona Innovación en donde bajo el paraguas del Consorcio de la Zona Franca de Barcelona se preveía crear un clúster de las industrias culturales, con participación de dinero público provenientes de las universidades públicas o las administraciones autonómica y local. Un proyecto que ha contado con el aval de conocidos consultores y empresarios de la gestión cultural (Marce, 2010). Estas propuestas de momento han sido aparcadas por falta de consenso y de recursos económicos, pero constituyen la constatación de que en política y gestión cultural se sigue apostando por proyectos de sostenibilidad y retorno social como mínimo dudosos y que, el sector académico y los consultores, en vez de criticar o moderar estas propuestas siguen impulsándolas como una forma de ganar reconocimiento o participación en proyectos y fondos. 
e innovadores en política cultural (Schuster, 2002; Rodriguez Morató, 2005). Por otra parte, también tienen importancia otros agentes que actúan en el contexto internacional. Sin embargo, estos organismos siguen anclados por inercia de formulaciones anteriores en el paradigma de la democratización de la política cultural. En el caso del Consejo de Europa la democratización cultural se articula en los derechos culturales y en la igualdad de acceso a las artes. Sin embargo, la Declaración de Friburgo en 2007 poco aporta de nuevo al panorama legitimador de las organizaciones internacionales.

Por otra parte, tal como señala Autissier (2013) la Comisión Europea no está aportando nuevas misiones o un refinamiento de las históricamente formuladas sino que cada vez se centra más en las lógicas de los operadores culturales, medios de comunicación y empresas tecnológicas. El discurso de la democratización cultural, largamente contrastado y refutado empíricamente por los consumos y hábitos culturales de los europeos pasa por sus horas más bajas, mostrando bajadas en los consumos en todos los países. ${ }^{12}$ La lógica de la oferta y la demanda u otras narrativas que tienen que ver más con la eficiencia y evaluación de las instituciones culturales tienden a sedimentarse en el discurso sobre la política cultural. Las recetas en forma de solución a la aporía de la democratización cultural por parte de algunos académicos franceses pasan por vincular la educación artística en el sistema educativo y dotar de recursos a las instituciones para que puedan dedicar las herramientas necesarias para fidelizar al mayor número de ciudadanos. Estas recetas francesas del mundo académico no tienen apenas eco en el contexto español, con un descenso pronunciado en los presupuestos públicos en cultura, una ley del mecenazgo que no termina de ser tramitada en el parlamento y una percepción de la corrupción política muy generalizada.

Asimismo, el discurso público sobre la cultura sigue centrándose en las grandes cifras públicas y el análisis de las ciencias sociales se centra en exceso en el análisis

${ }^{12}$ La disminución media de la Unión Europa (UE-27) alcanzó aproximadamente un punto, pasando del $9.6 \%$ al $8.8 \%$, entre 2001 y 2011; mientras que los datos por países muestran ligeras disminuciones de alrededor de 1 punto porcentual en prácticamente todos los casos de centro-Europa, pueden observarse caídas más acusadas en los países del sur como Grecia, Portugal y España. Este adelgazamiento del gasto cultural en el conjunto del gasto de las familias habría que situarlo pues en el contexto de una tendencia de largo recorrido, vinculada con la evolución de los hábitos de consumo, que con el impacto puntual y concreto de la crisis (Cf. Eurostat Estadísticas 2013, Disponible en:

$<$ http://epp.eurostat.ec.europa.eu/portal/page/portal/household_budget_surveys/introduction>).

Asimismo, el análisis comparado de la participación cultural en la Unión Europea nos ofrece por otra parte una idea de los retos de la política cultural en Europa y en España. Un informe publicado en el 2013 por la Comisión Europea (European Comission, 2013) que compara los hábitos culturales de los europeos de forma comparada en los años 2007 (antes de la crisis económico-financiera) y en 2013 concluye que el compromiso de los europeos con la actividad cultural disminuye a nivel general, incluso entre los programas de televisión y radio, los más populares. El estudio cartografía una Europa dividida en al menos dos bloques. Un bloque continental y nórdica en el que, a pesar de una leve bajada, se mantienen una alta tasa de actividad cultural. Y un bloque en el que los consumos y las prácticas culturales son bajos como el caso de la Europa del sur y del Este (ibídem). 
cuantitativo de las prácticas y consumos (Donnat, 2004). En este sentido, el discurso académico ha de proveer un nuevo sentido a las fotografías fijas de las encuestas, sabiendo que la cultura se asume más que se consume. Una de las alternativas propuestas a este paradigma se ha elaborado en Gran Bretaña y propone medir y potenciar el valor público de las propias instituciones culturales, es decir, el valor que la ciudadanía otorga a dicha institución cultural (Holden, 2004). Otra alternativa se centra en el valor de la política cultural en la adquisición de competencias, experiencias, valores y capacidad de reinvención y adaptación en un entorno de cambio social acelerado (Jones, 2010).

En este contexto de crisis de la política cultural, se ha convertido en un lugar común el mencionar el mecenazgo o el crowdfunding como una o la única tabla de salvación del sector cultural y como una forma de articular el apoyo social a la cultura, superando la tradicional tutela del Estado. Se trata de un discurso no sólo defendido por la derecha liberal sino que también ha sido incorporado al discurso del sector cultural y de los partidos de centroizquierda. Al margen del debate conceptual sobre el mecenazgo (a menudo se apela al mecenazgo privado sin atender que el dinero donado en parte se trata de recursos que el estado deja de ingresar y de administrar y por lo tanto son recursos públicos), los datos que nos ofrecen las experiencias internacionales son un jarro de agua fría en buena parte de las expectativas creadas (Rubio y Rius, 2014). En primer lugar, los ingresos por mecenazgo no son menos sino más pro-cíclicos que los ingresos públicos, es decir, aumentan más que los ingresos públicos en épocas de bonanza pero también tienden a disminuir con mayor velocidad y volumen que los ingresos públicos, comprometiendo así a los proyectos que dependan de sus ingresos (ibídem). En segundo lugar, el análisis de cómo se financia da como resultado la constatación de su concentración geográfica en la capital o las grandes ciudades y en las instituciones de la alta cultura que gozan de mayor visibilidad social o se convierten en un círculo social de las élites. Por lo tanto, el mecenazgo representa una vana esperanza para las organizaciones comunitarias de las regiones periféricas y plantea un debate del carácter regresivo a nivel social y desequilibrador a nivel regional de unos recursos que, repetimos, en parte son públicos. Y en tercer lugar, excepto en el caso de Francia en el que la ley de mecenazgo del 2003 ha conseguido un relativo éxito, en muchos países con una escasa tradición en este sentido, su evolución ha sido negativa y suponen una parte insignificante de los recursos. En definitiva, más que una tabla de salvación, el mecenazgo puede ser un "espejismo" que despiste más que oriente la salida de la crisis en el sector cultural (Rubio y Rius, 2014). ${ }^{13}$

${ }^{13}$ Las donaciones privadas Estados Unidos sufrieron ya en 2008 un descenso de cerca del $7 \%$-el primero desde 1998-, y a su vez, disminuyó de forma significativa el porcentaje de donaciones con finalidades culturales respecto el total de donaciones. El Reino Unido y Francia también registraron datos negativos ya desde el inicio de la crisis. Ambos países constituyen casos interesantes de análisis en Europa, el Reino Unido como ejemplo del modelo anglosajón, convencional, y Francia como nuevo modelo emergente de legislación sobre mecenazgo en Europa, gracias al marco normativo introducido en 2003. Precisamente en este país, las donaciones por mecenazgo empresarial según datos de ADMICAL (asociación francesa de 
No deja de ser preocupante que una parte de los expertos consultados asuman la renuncia del Estado a garantizar el acceso de todos los ciudadanos a los servicios públicos y la misión del Estado de redistribuir la riqueza y los bienes, entre ellos los culturales. Y que se asuma, de forma naturalizada, o taked for granted en la expresión anglosajona, que las administraciones públicas no puedan en el futuro ejercer este rol. Asimismo, debemos ser conscientes de que el mecenazgo y otras formas de financiación pueden ser utilizados como una "salida neoliberal" (reducción del Estado) a la crisis, al responsabilizar a las empresas y al ciudadano de la financiación de la cultura. Sin embargo, por la experiencia de otros países y por la estructura del mecenazgo actual podemos suponer que este mecenazgo se dirigiría a las grandes instituciones de la alta cultura radicadas en las grandes ciudades, aumentando así de facto la elitización de la cultura.

En relación al crowdfunding, es una realidad aún joven y como hemos podido analizar en la investigación realizada (Rubio Arostegui et al., 2014) no se dispone aún de muchos datos ni estudios longitudinales, sabemos solamente que genera mucho entusiasmo y a la vez que representa una parte muy pequeña de la financiación de la cultura. ${ }^{14}$ Por otra parte, empieza a generar algunas dudas sobre su naturaleza:¿Se trata de una pre-venta o de un encargo más que del mecenazgo clásico? ¿Para qué tipo de producciones culturales es adecuado y cuáles no? ¿Se puede emplear en proyectos que reciban financiación pública o que sean promovidos por las administraciones públicas? ¿Puede generar proyectos estables o bien contribuye a una mayor precarización e inestabilidad del trabajo artístico? Existen demasiados interrogantes abiertos como para poderlo considerar en la actualidad una solución al nivel que algunos autores o agentes culturales lo sitúan en el marco del Delphi realizado. Las ciencias sociales deben mostrarse pues críticas con los discursos dominantes y también con los discursos utópicos en relación

promoción del mecenazgo) disminuyeron un $20 \%$ entre 2008 y 2010 pasando de 2.5 a 2 millardos de euros. Pero esto no es lo más significativo: la cultura fue el sector que anotó una mayor bajada, pasando de 975 millones de euros en 2008 a tan solo 380 millones de euros en 2010, de forma que las donaciones para finalidades culturales sufrieron un severo recorte, quedando reducidas a menos de la mitad en tan solo 2 años y pasando del 37\% del total recaudado a tan solo el 19\% (Rubio Arostegui et al., 2014).

${ }^{14}$ Un estudio realizado por una consultoría y citado por la Comisión Europea, cifra en 735 millones de euros el total de lo recaudado mediante este procedimiento en todos los sectores, sin especificar que montante corresponde a cultura. Asimismo, el informe también afirma que respecto al 2011 el crecimiento ha sido de un $65 \%$, a pesar del contexto de crisis. No obstante, el mismo informe reconoce que tratándose de cifras conjuntas para toda la Unión Europea, se trata de cifras modestas (Massolution, 2013). Otro estudio centrado en España (Infocrowdsourcing) y Latinoamérica, ofrece datos para España: 12,9 millones de euros recaudados por las plataformas de crowdfunding en España el 2009. De este total, un 10\% fueron a parar a proyectos de música (1,3 millones) y un 13\% a las artes (1,7 millones). Sin embargo, no poseemos datos ni estudios de los outputs y outcomes de estas iniciativas: cuántos proyectos se terminaron, cuántos espectadores los vieron, qué valoraciones y premios recibieron estas iniciativas o qué dinámicas culturales o sociales generaron. 
a la cultura para contrarrestar su tendencia a favorecer la privatización de este derecho social y a la vez mostrar caminos alternativos a estas tendencias.

\subsection{Acompañar la modernización de la gobernanza y la gestión de la cultura}

Como hemos comentado anteriormente, en los últimos treinta años el análisis de la gestión cultural se ha basado en un estudio, habitualmente celebratorio, de los éxitos de los proyectos culturales, en una perspectiva que examinaba la eficacia cultural en bruto en términos de usuarios o espectadores y últimamente, su impacto económico. Esta perspectiva dejaba de lado toda consideración del coste-beneficio, de la eficiencia en alcanzar tales metas o bien, finalmente, la coherencia con los objetivos de carácter público de las inversiones públicas en cultura y el impacto a largo plazo en los sectores culturales y la ciudadanía. Un punto de vista que ha favorecido como hemos dicho la proliferación de agencias e instituciones culturales públicas o semi-públicas y de iniciativas privadas sin mecanismos de control o tutela (Rius-Ulldemolins, 2014). Sin embargo, los contratos programa han supuesto una oportunidad para poner remedio a este déficit. Esta fórmula de gestión ha tenido su aplicación en Gran Bretaña, Flandes o Francia y que ha representado un paso adelante en los procesos de agencialización y contractualización de las relaciones entre las administraciones públicas y las instituciones culturales. Sin embargo, los contratos programa u otras fórmulas de modernización de la gestión pública han tenido una aplicación más bien escasa en España, con la excepción de Cataluña (Rius y Rubio, 2013).

Sin embargo, con la llegada de la crisis del 2008, las administraciones públicas, siguiendo el consejo de ciertos expertos de orientación neoliberal, han considerado que existía una burbuja cultural y han apostado por primar el criterio de la eficiencia, por encima de cualquier otra consideración y situando la llamada cooperación público-privada como la solución a la retirada del Estado (Cf., Colomer, 2011). A causa de ello se han eliminado las agencias y se han internalizado de nuevo muchas estructuras e iniciativas culturales, que han abortado los tímidos avances en los procesos de modernización de la gestión (agencialización, gerencialización, contratos programa) fomentando otra vez la burocratización de la gestión cultural en contra de toda la experiencia acumulada en gestión cultural que recomendaba lo contrario (Rius y Rubio, 2013). En este caso, la eficiencia no ha sido un valor que haya predominado en la acción pública. En el marco de la "burbuja cultural" no se desarrollaron los instrumentos de planificación (con algunas excepciones) que limitaran las inversiones y sus dimensiones introduciendo el valor de la eficiencia y la sostenibilidad (Martinez y Rius, 2010) ${ }^{15}$ y ahora,

${ }^{15}$ Una de las pocas experiencias de planificación de la inversión en infraestructuras culturales ha sido el Plan de Equipamientos Culturales de Cataluña (2010-2020) elaborado inicialmente por Santi Martinez Illa que intenta delimitar con criterios objetivos y participativos la dotación y dimensión de los equipamientos culturales, especialmente, escénicos y musicales, los centros de arte y los centros culturales poliva- 
en el marco "del recorte", no hay una evaluación para tomar decisiones en virtud de este valor (Rubio y Rius, 2012).

Por otra parte, en el ámbito de la gobernanza cultural las ciencias sociales no han hecho grandes aportaciones, a pesar de que desde hace veinte años se viene destacando que la creciente interrelación entre los diferentes ámbitos culturales, entre el sector público y el sector privado y las cada vez mayores intersecciones entre ámbitos culturales y creativos, aconsejan crear dispositivos de gobernanza orientados a la generación de valor cultural y público (Rius, 2005). Sin embargo, los mayores progresos en España se han realizado solamente en el ámbito de la planificación estratégica en cultura desde los años noventa (Manito, 2008), que imitaba las prácticas iniciadas en el Reino Unido (Evans, 2001). Este proceso seguía la estela del modelo de planificación posfordista iniciado en otros campos de las políticas públicas, en un momento en que un entorno más inestable a nivel socio-económico, la reforma del sector público y las tendencias descentralizadoras recomiendan adoptar este tipo de planificación más flexible (Harvey, 1989; Lash y Urry, 1998). En cultura, esta planificación estratégica deriva hacia una proliferación de la planificación estratégica de ámbito estrictamente local (a pesar de algunos intentos de ampliarlo a la zona metropolitana) y el abandono de todos los intentos de planificación regional basados en la satisfacción de derechos culturales básicos y la corrección de las desigualdades en accesibilidad cultural a nivel autonómico o estatal (Martinez y Rius, 2010; Rius-Ulldemolins et al., 2012).

\subsection{Actualizar y buscar consensos en la relación entre la industria cultural, el sector cultural y los movimientos sociales en torno a los derechos de autor y la creación digital}

Como hemos dicho, a partir de la segunda mitad de la década de los años noventa el enfoque de la planificación estratégica en la cultura parecía dotar de un aire renovador a la toma de decisiones sobre la cultura y avanzar hacia una gestión más integral e incluyente de la cultura, desde los sectores industriales a los profesionales, pasando por los amateurs (Rius, 2014). Sin embargo, en la práctica, su función en términos participativos ha sido muy limitada a los sectores industriales y profesionales $\mathrm{y}$, con ciertas

lentes (Martinez y Rius, 2010). En este sentido, hay que revisar de forma crítica la llamada planificación estratégica cultural, que se ha desarrollado por todo el territorio. Este tipo de planificación ha representado a menudo una retórica legitimadora de grandes proyectos insostenibles en función de oportunidades a corto plazo y no una planificación racional en función de las necesidades sociales y culturales reales y a largo plazo. En el caso de las políticas culturales, como en otras áreas, los gastos culturales se encuentran hipotecados por la amortización y los costes de funcionamiento resultado de proyectos de infraestructuras culturales -que representan aproximadamente una cuarta parte de los gastos culturales totales- y que no disponen de un plan de actividades, ni previeron una dotación suficiente para gastos de personal o de mantenimiento, que conforma la mayor parte del gasto de los equipamientos culturales (Ministerio de Educación y Cultura, 2013). 
restricciones en la toma de decisiones, sin llegar a constituirse como escenarios deliberativos respecto de los diversos actores y sectores involucrados (Sánchez et al., 2013).

En este contexto ha irrumpido el paradigma digital, al que estos mecanismos de gobernanza no han sabido dar una respuesta, desarrollándose un distanciamiento muy importante entre los sectores culturales y la sociedad, en especial en relación a los consumos digitales y los derechos de autor, un fenómeno al que las ciencias sociales no han sabido interpretar ni paliar adecuadamente. Así pues, los derechos de autor se han convertido en uno de los temas más controvertidos en los que se desarrollan discursos contrapuestos y que tienen una notable dimensión política y un impacto en el debate público sobre la cultura (Levine et al., 2013). Hasta la actualidad se han desarrollado dos tipos de discursos muy polarizados en torno de la propiedad -como se ha podido comprobar en el Delphi y en los grupos de discusión realizados en el marco de la investigación sobre financiación de la cultura (Rubio Arostegui et al., 2014)-, una entendiéndola como un derecho inalienable y como la base económica del sistema cultural y otro, interpretándola como un abuso y apropiación de unos pocos de una propiedad común, el conocimiento y la cultura (Sábada, 2008). En este contexto, en algunos países europeos se ha intentado encontrar soluciones intermedias que formen un punto de encuentro entre estas dos posiciones como la generación de licencias únicas para la Unión Europea (Psychogiopoulou, 2009) o bien las tarifas planas culturales (Media Consulting Group, 2011). Unas medidas que ni tan siquiera han sido estudiadas en el caso español.

En este contexto, mientras las ciencias sociales en general se focalizaban hacia un análisis de las dimensiones y los impactos económicos de la cultura, han surgido fenómenos culturales que escapan a la comprensión en términos de mercado o de acción redistributiva del Estado y que han sido en buena medida interpretados como un resurgimiento de las dinámicas de intercambio que analiza Polanyi (2006). Es sin duda en estos ámbitos de construcción de un común cultural en los que se están desarrollando los proyectos más innovadores y que requieren una renovada atención por parte de las ciencias sociales (Ariño Villarroya, 2009; Subirats et al., 2011). Actualmente, podemos observar la proliferación de nuevas formas de creación cooperativa desarrolladas en buena medida a partir de internet - pero no solamente circunscritas en este ámbito- y que permiten reconectar a las nuevas generaciones nacidas en el paradigma digital a la cultura y a la creación. Asimismo, también están apareciendo iniciativas de abajo-arriba (bottom-up) que mantienen espacios colaborativos trandisciplinares (coworking) y de creación y fabricación (fablab). Unos espacios que facilitan las dinámicas de polinización cruzada entre sectores culturales y creativos características de las nuevas dinámicas culturales de la modernidad avanzada (Scott, 2007; Currid y Williams, 2010). Estos fenómenos, en los que confluyen innovación social y cultural, constituyen evidentes islas de esperanza frente a las sucesivas oleadas neoliberales y el colapso de los sistemas de creación y difusión, en los que se ha basado la modernidad cultural, debe 
servir de estímulo a los científicos sociales para repensar el rol de la esfera cultural y el valor que aporta a la sociedad.

\section{Conclusiones}

El sector cultural ha vivido estas dos últimas décadas en España una fase de gran expansión seguida de un "diluvio" que ha conllevado a una situación de parálisis al sistema cultural. Asimismo, la emergencia del paradigma digital ha conllevado la aparición de una crisis sistémica, a la que los sectores culturales tienen dificultad de afrontar de una forma ordenada. Frente a esta situación, las ciencias sociales no han sabido articular un análisis crítico a las instrumentalizaciones de la cultura durante el periodo de expansión ni tampoco actualmente están sabiendo dar respuesta a los retos actualmente planteados.

Por el contario, tal y como hemos mostrado en este artículo, las ciencias sociales en España, sea desde su ámbito académico puro o bien desde el ámbito aplicado de la consultoría han ejercido de legitimadores de las perspectivas -como la ciudad creativa o el impacto social y económico de la cultura- que han fomentado los excesos y la desvinculación en relación a objetivos de valor cultural. Tampoco ha contribuido a una crítica de los mecanismos de gobernanza y planificación a pesar de sus evidentes disfunciones e ineficiencias. Con ello, la crisis ha impactado de lleno en la política cultural sin que desde las ciencias sociales se haya sabido responder o analizar al menos hasta el momento los elementos para re-articular el sistema cultural o buscar nuevos objetivos y legitimaciones.

De este modo, las ciencias sociales deben reivindicarse como un ámbito fundamental para refundar la política social en un escenario post-ciudad creativa, en el que la reivindicación de la autonomía y la cultura debe basarse en una constatación rigurosa de los efectos cohesionadores de las prácticas y los consumos culturales. Asimismo, las ciencias sociales deben abordar críticamente las iniciativas culturales instrumentalizadoras, así como los discursos utópicos o las falsas salidas a las crisis señalando las debilidades socio-económicas o sus efectos no deseados y no solamente las promesas de futuros beneficios y efectos positivos. El soporte a la acción pública no debe por lo tanto entenderse como una legitimación de todas las políticas culturales sino que debe estudiarse cuáles son eficaces, eficientes y aportan valor público y cuáles no, evitando así escenarios en el que el "diluvio" de la reducción de ingresos públicos y privados de los sectores culturales se suma a una debilidad sistémica en su gobernanza y sostenibilidad económica, social y cultural.

En este artículo, creemos haber empezado a señalar algunos caminos por los que las ciencias sociales pueden dar respuesta y ejercer así su responsabilidad en el devenir de la esfera cultural. Los autores proponemos tres líneas de investigación por las que se debe avanzar en el futuro próximo: 1) Formas de relegitimación de la cultura a partir de 
nuevas formas de creación de valor público de la cultura. 2) Nuevas formas de gobernanza y gestión más eficientes de la cultura y que antepongan el valor cultural y el retorno social a largo plazo a otras consideraciones de tipo instrumental, y 3) Dinámicas y mecanismos de participación cultural e innovación social en el ámbito de la creación digital colaborativa, así como nuevas formas de regulación de los derechos de autor acordes con estas realidades. En definitiva, las ciencias sociales en el ámbito de la cultura deben dejar de legitimar la reconversión neoliberal de esta esfera al servicio de las élites políticas y las grandes empresas para empezar a imaginar nuevos escenarios de sostenibilidad y valor público para cuando amaine el "diluvio".

\section{Bibliografía}

Ariño Villarroya, A. (2010): Prácticas culturales en España:desde los años sesenta hasta la actualidad, Barcelona, Ariel.

Ariño Villarroya, A. (2009): El Movimiento Open:la creación de un dominio público en la era digital, València, Universitat de València.

Autissier, A.M. (2013): "Postface: Quels contenus pour la démocratisation culturelle dans l'Europe du XXIe siècle ?", en Philippe Poirrier, ed. Démocratiser la culture! Une histoire comparée des politiques culturelles. Territoires contemporaine, Dijon, Centre Georges Chevrier, Université de Bourgogne, pp. 126-134.

Balibrea, M.P. (2004): "Barcelona: del modelo a la marca", en Jesús Carrillo, Ignacio Estella Noriega y Lidia García-Merás, eds. Desacuerdos 3. Sobre arte, políticas y esfera pública en el estado Español, Barcelona, Arteleku -MACBA- Universidad Internacional de Andalucía, pp. 261-271.

Belfiore, E. \& O. BennetT (2008): The Social Impact of the Arts: An Intellectual History, Basingtoke, Palgrave/Macmillan.

Belfiore, E. (2004): "Auditing Culture. The subsidised cultural sector in the New Public Management", International Journal of Cultural Policy, 10, pp. 183-202.

Belfiore, E. (2002): "Art as a means of alleviating social exclusion: Does it really work? A critique of instrumental cultural policies and social impact studies in the UK", International Journal of Cultural Policy, 8, pp. 91-106.

Belfiore, E. \& O. BenNetT (2007): "Rethinking the social impact of the arts", International Journal of Cultural Policy, 13, pp. 135-151.

BIANCHINI, F. (1991): City centres, city cultures:the role of the arts in the revitalisation of towns and cities, London, Centre for local economic strategies.

Bilton, C. (2007): Management and creativity:from creative industries to creative management, Malden, MA, Oxford, Blackwell Pub. 
Bloomfield, J. \& F. Bianchini (2004): Planning for Intercultural City. London, Comedia.

Bourdieu, P. (2002): Las Reglas del arte:génesis y estructura del campo literario, 3a edn,. Barcelona, Anagrama.

Bourdieu, P. (1991): La distinción: criterios y bases sociales del gusto, Madrid, Taurus.

BouRdieu, P. (1977): "La production de la croyance: contribution à une économie des biens symboliques", Actes de la recherche en sciences sociales, 13, pp. 3-43.

Bourdieu, P., A. Darbel y D. Schnapper (2003): El amor al arte: los museos europeos y su público, Barcelona, Paidós.

BouZADA, X. (2008): "La gobernabilidad multinivel da la cultura en España” en Antonio Albino Canelas Rubim y Bayardo Rubens, eds. Politicas Culturais Na Ibero-América, Salvador, Editora da Universidade Federal da Bahia, pp. 159-200.

Bustamante, E. (2011): "Industrias culturales, economía creativa y sociedad" en Luis A. Albornoz, ed. Poder, medios, cultura. Una mirada crítica desde la economía política de la comunicación, Buenos Aires, Paidós, pp. 139-156.

Colomer, J. (2011): Oportunitats de la cooperació público-privada en la gestió de la cultura, Barcelona, IESE Business School, Centro Sector Público - Sector Privado.

Cunningham, S. (2009): "Trojan horse or Rorschach blot? Creative industries discourse around the world", International Journal of Cultural Policy, 15, pp. 375-386.

CURRID, E. \& S. Williams (2010): “The geography of buzz: art, culture and the social milieu in Los Angeles and New York", Journal of Economic Geography, 10, pp. 423-451.

Degen, M. \& M. García (2012): "The Transformation of the Barcelona Model?: An Analysis of Culture, Urban Regeneration and Governance", International Journal of Urban and Regional Research, 36, pp. 1022-1038.

Delgado, M. (2008): "La artistización de las políticas urbanas. El lugar de la cultura en las dinámicas de reapropiación capitalista de la ciudad”, Scripta Nova: revista electrónica de geografia y ciencias sociales, 12.

Dinnie, K. (2008): Nation Branding Concepts, Issues, Practice, Oxford, ButterworthHeinemann.

Donnat, O. (2004): "Les univers culturels des Français", Sociologie et societés, pp.36-1 y $87-103$.

Dubois, V. (2010): "Le modele français et sa crise: ambitions, ambigüités et défis d'une politique culturelle" en Diane Saint-Pierre y Claudine Audet, eds. Tendances et défis des politiques culturelles, Québec, Presses de l'Université de Laval, pp. 17-52.

Dubors, V. (1999): La politique culturelle. Genèse d'une catégorie d'intervention publique, Paris, Belin. 
Dubois, V., Cl. Bastien, A. Fryermuth, \& K. Matz (2012): La politique, l'artiste et le gestionnaire. (Re)configurations locales et (dé)polisation de la culture, Bellecombeen-Bauges, Éditions du Coquant.

Ema López, J.E. (2013): "Qué habría sido de las ciencias sociales", Educatio Siglo XXI: Revista de la Facultad de Educación, 31, pp. 15-34.

European Comission (2013): Cultural Access and Participation. Brusselas, European Comission.

Evans, G. (2001): Cultural Planning: An urban renaissance?, Londres, Routledge.

Evans, G. (2003): "Hard-Branding the cultural city-from Prado to Prada", International Journal of Urban and Regional Research, 27, pp. 417-440.

Fishman, R.M. \& O. Lizardo (2013): "How Macro-Historical Change Shapes Cultural Taste: Legacies of Democratization in Spain and Portugal", American Sociological Review, 78, pp. 213-239.

FloRIDA, R.L. (2005): Cities and the creative class, Londres, Routledge.

FulLerton, L. \& J. EtTema (2014): "Ways of worldmaking in Wikipedia: reality, legitimacy and collaborative knowledge making", Media, Culture \& Society, 36, pp. 183-199.

GarcíA, B. (2004): "Cultural Policy and Urban Regeneration in Western European Cities: Lessons from Experience, Prospects for the Future", Local Economy, 19, pp. 312-326.

GRAY, C. (2008): "Instrumental policies: causes, consequences, museums and galleries", Cultural Trends, 17, pp. 209-222.

Gray, C. (2007): "Commodification and instrumentality in cultural policy", International Journal of Cultural Policy, 13, pp. 203-215.

Harvey, D. (1989): "From Managerialism to Entrepreneurialism: The Transformation in Urban Governance in Late Capitalism", Geografiska Annaler. Series B, Human Geography, 71, pp. 3-17.

HernÀndez i Martí, G-M. y M. Albert Rodrigo (2012): "La dinámica general de la política cultural en el País Valenciano: posiciones, discursos y prácticas de los actores culturales valencianos", Revista de Investigaciones Políticas y Sociológicas, 11, pp. 89-114.

Holden, J. (2008): Democratic Culture. Opening Up the Arts for Everyone, London, Demos.

Holden, J. (2004): Capturing cultural value, London, Demos.

Jones, S. (2010): Culture Shock, London, Demos.

Kagan, S. \& J. HaHn (2011): "Creative cities and (Un)Sustainability: From Creative Class to Sustainable Creative Cities", Culture and Local Governance, 3, pp. 1-2 y 11-27.

Lage, X., A. Losada y M. Gómez (2012): "La política cultural en la comunidad autónoma gallega: de la dependencia a la autonomía", Revista de Investigaciones Políticas y Sociológicas, 11, pp. 115-148. 
LANDry, Ch. \& F. BiAnchini (1995): The creative city, Great Britain, Demos.

Lash, S. y J. URry (1998): Economías de signos y espacio: Sobre el capitalismo de la posorganización, Buenos Aires, Amorrortu.

Levine, R., F. Caballero y V. Campos (2013): Parásitos:cómo los oportunistas digitales están destruyendo el negocio de la cultura, Barcelona, Ariel.

Majoor, S. (2011): "Framing Large-Scale Projects: Barcelona Forum and the Challenge of Balancing Local and Global Needs", Journal of Planning Education and Research, 31 , pp. 143-156.

Manito Lorite, F. (ed) (2010): Ciudades Creativas, Fundación Kreanta, Barcelona.

Manito Lorite, F. (2008): Planificación estratégica de la cultura en España, Madrid, Fundación Autor.

Marce, X. (2010): "Eje cultural. Barcelona Zona Innovació” en Consorci de la Zona Franca, ed. El projecte empresarial Barcelona Zona Franca, Barcelona, Consorci de la Zona Franca, pp. 16-20.

Martinez, S. \& J. Rius (2010): “Cultural planning and community sustainability: The case of the Cultural Facilities Plan of Catalonia (PECCAT 2010-20", Culture and Local Goverment, 3, pp. 1-2 y 71-82.

Massolution (2013): Crowdfunding Industry Report, Los Angeles, Massolution.

McGuigan, J. (2004): Rethinking cultural policy, Maidenhead, Open University.

Media Consulting Group (ed) (2011): The "Content Flat-Rate": A Sollution to Illegal FileSharing, European Parliament, Brussels.

Menger, P.-M. (2010): Cultural policies in Europe. From a state to a city-centered perspective on cultural generativity, Tokyo, National Graduate Institute for Policy Studies.

Miles, M. (2005): "Interruptions: Testing the Rhetoric of Culturally Led Urban Development", Urban Studies, 42, pp. 889-911.

Ministerio de Educación y Cultura (2013): Programa Platea. Programa estatal de artes escénicas, Madrid.

Mooney, G. (2004): "Cultural Policy as Urban Transformation? Critical Reflections on Glasgow, European City of Culture 1990”, Local Economy, 19, pp. 327-340.

PeCK, J. (2005): "Struggling with the Creative Class", International Journal of Urban and Regional Research, 29, pp. 740-770.

PIKE, A. (2011): Brands and branding geographies, Cheltenham, Northampton, Edward Elgar.

Poirrier, P. (2013): “Une histoire comparée de la démocratisation culturelle”, Territoires contemporains, 5, p. 10. 
Polanyi, K. y J.E. Stiglitz (2006): La Gran transformación:los orígenes políticos y económicos de nuestro tiempo, México, Fondo de Cultura Económica.

Pratt, A.C. (2008): "Creative cities: the cultural industries and the creative class", Geografiska Annaler: Series B, Human Geography, 90, pp. 107-117.

Psychogiopoulou, E. (2009): Les sociétés de gestion collective et la diversité culturelle dans le secteur de la musique, Parlement européen, Bruxelles.

Rius-Ulldemolins, J., A. Rodríguez Morató y S. Martinez Illa (2012): "El sistema de la política cultural en Cataluña: un proceso inacabado de articulación y racionalización", Revista de Investigaciones Políticas y Sociológicas, 11, pp. 173-204.

Rius-Ulldemolins, J. \& M.M. Zamorano (2014): "Spain's nation branding project Marca España and its cultural policy: the economic and political instrumentalization of a homogeneous and simplified cultural image", International Journal of Cultural Policy,: pp. 1-21.

Rius, J. (2014): "La gobernanza y la gestión de las instituciones culturales nacionales: de la oposición entre arte y economía a la articulación entre política cultural y management", Revista Papers, 99, pp. 73-95.

Rius, J. (2005): Un nou paradigma de la política cultural. Estudi sociologic del cas Barcelona, Barcelona, Universitat Autònoma de Barcelona - École des Hautes Études en Sciences Sociales.

Rius, J. \& A. RuBio (2013): "The governance of national cultural organisations: comparative study of performance contracts with the main cultural organisations in England, France and Catalonia", International Journal of Cultural Policy, 19, pp. 249-269.

Rius-Uldemollins, J. y M.M. Zamorano (2014): “¿Es España un estado casi-federal en política cultural? Articulación y conflicto entre la política cultural de la Administración General del Estado y de la Comunidad Autónoma de Cataluña", Revista d'Estudis Autonòmics i Federals, 19, pp. 274-309.

Rius-Ulldemolins, J. (2014): "Modelos de política cultural y modelos de equipamientos culturales: de los modelos nacionales a los modelos locales. Análisis del caso de Barcelona", Política y Sociedad, 51, pp. 399-422.

Rodriguez Morató, A. (2012): "El análisis de la política cultural en perspectiva sociológica. Claves introductorias al estudio del caso español", Revista de Investigaciones Políticas y Sociológicas, 11, pp. 15-38.

Rodriguez Morató, A. (2005): "La reinvención de la política cultural a escala local: el caso de Barcelona", Sociedade e Estado, 20, pp. 351-376.

Rodríguez Morató, A., X. BouZada y A. Ariño (2005): "La política cultural en España” en Juan Antonio Roche Cárcel y Manuel Oliver Narbona, eds. Cultura y globalización. Entre el conflicto y el diálogo, San Vicent del Raspeig, Publicaciones de la Universidad de Alicante, pp. 435-472. 
Roselló, D. y A. Colombo (eds.) (2008): Gestión Cultural: Estudios de caso, Ariel, Barcelona.

Rowan, J. (2010): Emprendizajes en cultura. Discursos, instituciones y contradicciones en la empresarialidad cultural, Madrid, Traficantes de Sueños.

Rubio Aróstegui, J.A. (2008): "Niveles de discurso de la política cultural y sus interacciones en la construcción de la realidad artística y cultural. El papel del discurso científico en España", Periférica, 9, pp. 21-40.

Rubio Arostegui, J.A., J. Rius-Ulldemollins y S. Martinez Illa (2014): El modelo español de financiación de las artes y la cultura en el contexto europeo. Crisis económica, cambio institucional, gobernanza y valor público de la cultura y la política cultural, Madrid, Fundación Alternativas.

Rubio, A. y J. Rius (2012): “La modernización de la gestión pública de la cultura. Análisis comparado del caso de los equipamientos culturales de las comunidades autónomas de Cataluña y Madrid", Gestión y Análisis de Políticas Públicas, 8, pp. 79-92.

Rubio, A., J. Rius y S. Martinez (2014): El modelo español de financiación de las artes y la cultura en el contexto europeo. Crisis económica, cambio institucional, gobernanza y valor público de la cultura y la política cultural, Madrid, Fundación Alternativas.

SÁbadA, I. (2008): Propiedad intelectual: ¿bienes públicos o mercancías privadas?. Madrid, Los libros de la catarata.

SÁnchez, M.V., J. Rius-Ulldemolins y M. Zarlenga (2013): “¿Ciudad creativa y ciudad sostenible?: Un análisis crítico del modelo Barcelona de políticas culturales", Revista Crítica de Ciências Sociais, 96, pp. 48-57.

Schlesinger, P. (2013): "Expertise, the academy and the governance of cultural policy", Media Culture Society, 35, pp. 27-35.

Schuster, J.M. (2002): "Sub-national cultural policy - Where the action is: mapping state cultural policy in United States", International journal of cultura policy, 8, pp. 181196.

Scott, A. (2007): "Capitalism and Urbanization in a New Key? The Cognitive-Cultural Dimension", Social Forces, 85, pp. 1465-1482.

Subirats, J., N. Barbieri y A. Partal (2010): "El retorn social de les polítiques culturals: de l'impacte social al valor públic", Quaderns d'Acció Social i Ciutadania, 10, pp. 18-24.

Subirats, J., X. Fina, N. Barbieri y E. Merino (2011): Proximitat, cultura i tercer sector a Barcelona. Barcelona, Icaria.

Urfalino, P. (1996): L'invention de la politique culturelle, Paris, La Documentation Française.

Urfalino, P. (1989): "Les politiques culturelles: mécénat caché et académies invisibles", L'Anne sociologique, 3, pp. 81-109. 
Zimmer, A. \& S. Toepler (1996): "Cultural Policies and the Welfare State: The Cases of Sweden, Germany, and the United States", The Journal of Arts Management, Law and Society, 26, pp. 167-193 